\title{
Recognition of post-MRCPsych training undertaken in the United Kingdom, by the Royal Australian and New Zealand College of Psychiatrists
}

As a result of discussions and correspondence with the Royal Australian and New Zealand College of Psychiatrists, additional recognition of training in psychiatry in the United Kingdom has been agreed by the RANZCP. The following are the RANZCP's agreed exemptions although it is emphasised that this scale should be used for guidance only and that each case will be individually considered on application to the Fellowships Board of the RANZCP. Experience has shown us that trainees who are considering working in Australia or New Zealand should pay particular attention during general professional training to the requirement of the RANZCP that trainees should spend six months in child psychiatry.

Before members commit themselves to working abroad they should check their position with respect to immigration and employment laws.

UK qualifications and experience

Recent acquisition of MRCPsych and some postMRCPsych experience.

MRCPsych plus significant senior registrar experience in the UK or Ireland.

MRCPsych plus more than three years as senior registrar and/or consultant appointment in the NHS.

\section{RANZCP exemptions}

Training to be assessed carefully, paying particular attention to whether the mandatory rotations, especially in child, liaison and chronic psychiatry, have been completed, in order for a Certificate of Accreditation to be issued.

Generally not exempt from the elective year.

Generally exempt from the case histories, but required to do written papers and Days 1 and 2 (Section 1).

Required to complete dissertation (Section 2).

Training to be assessed carefully. If mandatory experiences have been completed, a Certificate of Accreditation will be issued.

Generally exempt from case histories; possible exemption from other parts of the examination, depending on experience (written paper 1 or both written papers and/or Day 2).

May be entitled to exemption from the elective year requirement.

Generally required to complete dissertation.

Generally eligible for a Certificate of Accreditation provided the mandatory training requirements have been satisfied.

Generally entitled to exemption from the elective year requirement.

Generally exempt from all the examination requirements, except Day 1 (Section 1 ).

May still be required to complete dissertation depending on publication record and higher degree, or other evidence of scholarship.

It should be noted, by those who wish to undertake some of their psychiatric training abroad, and possibly return to the UK in the future to practice psychiatry, that in order to be eligible for "T" after their name on the GMC's register, indicating completion of specialist training, four years higher training in the UK or appointment to a consultant post in the NHS are required.

June 1991

Dr Fiona Caldicott

Dean 